\section{EVOLUCIÓN DE LA CULTURA DE DEFENSA EN LA ÚLTIMA DÉCADA}

\author{
Fernando Moréu Munáiz \\ 2o Comandante B/E "Juan Sebastián de Elcano" \\ fmormu3@oc.mde.es
}

Cómo citar este artículo/Citation: Moréu Munáiz, F. (2014). "Evolución de la cultura de defensa en la última década". Arbor, 190 (765): a095. doi: http://dx.doi.org/10.3989/ arbor.2014.765n1002

Recibido: 5 junio 2012. Aceptado: 2 diciembre 2013.

RESUMEN: El IEEE (Instituto Español de Estudios Estratégicos) impulsa, desde 1997 por medio del CIS (Centro de Investigaciones Sociológicas), la encuesta "La Defensa Nacional y las Fuerzas Armadas". En ella se recoge, entre otras cuestiones, la opinión de los españoles en asuntos relacionados con el sentimiento y compromiso de sentirse español, la percepción de vivir en un mundo inseguro o el grado de reconocimiento de la sociedad española hacia sus Fuerzas Armadas. El estudio de la serie temporal de sus datos permite valorar el apoyo de los ciudadanos a sus ejércitos y evaluar la eficacia de los esfuerzos dedicados a ello. El análisis conjunto de los datos muestra una sociedad española con una elevada opinión de sus Fuerzas Armadas pero con un menor compromiso hacia los valores que estas representan y con un creciente interés para que su presupuesto se ajuste al menor presentimiento de amenazas militares contra España. La característica más notable de la sociedad española hacia sus Fuerzas Armadas es su enorme heterogeneidad cuando se segmenta por su edad, por su comunidad autónoma o por su ideología política.

PALABRAS CLAVE: Fuerzas Armadas; cultura de defensa; Defensa Nacional; estudios sociológicos.

\section{EVOLUTION OF THE CULTURE OF DEFENCE OVER THE LAST DECADE}

Copyright: (C) 2014 CSIC. Este es un artículo de acceso abierto distribuido bajo los términos de la licencia Creative Commons Attribution-Non Commercial (by-nc) Spain 3.0.

ABSTRACT: Since 1997, the IEEE (Spanish Institute for Strategic Studies) has commissioned the CIS to conduct its "National defence and armed forces" survey. This contains, inter alia, the opinion of the Spanish public on matters related to the feeling and commitment to 'Spanishness', the perception of living in an insecure world or the degree of recognition Spanish society gives to its armed forces. The study of the time series of data allows public support for the armed forces to be assessed and to evaluate the effectiveness of efforts to bolster it. An overall analysis of the data shows a Spanish society with a highly positive opinion of its armed forces but with a diminished commitment to the values that they represent, a growing concern for its budget to be smaller, and a reduced sense of external threats. The enormous heterogeneity is the most notable feature of the Spanish society towards its armed forces and it is segmented by age, autonomous community and political ideology.

KEYWORDS: Armed Forces; culture of defence; National Defence; opinion survey. 
Las dos últimas Directivas de Defensa Nacional $1 / 2008$ y $1 / 2012$, señalan la necesidad de fomentar la cultura de seguridad y defensa en la sociedad, promoviendo el más amplio apoyo de los ciudadanos a sus ejércitos que concite la plena identificación de la sociedad con sus Fuerzas Armadas y subrayando que la defensa de España debe ser asumida por todos los españoles como asunto de indudable trascendencia.

Este reconocimiento de la sociedad con sus Fuerzas Armadas significa conseguir que los asuntos que tienen que ver con la seguridad, con la defensa, con los ejércitos y los militares, no sean ajenos a los ciudadanos.

La política de comunicación del Ministerio de Defensa promueve que la sociedad conozca a sus Fuerzas Armadas y, de esta manera, se identifique con ellas. Los medios de comunicación social difunden este mensaje.

Para evaluar el éxito del mismo y determinar así el grado de cultura de defensa en la sociedad española, el Instituto Español de Estudios Estratégicos (IEEE) impulsa desde 1997 -por medio del Centro de Investigaciones Sociológicas (CIS)- una encuesta, denominada "La Defensa Nacional y las Fuerzas Armadas", a través de entrevistas personales en domicilios de toda España excluyendo las ciudades autónomas de Ceuta y Melilla ${ }^{1}$.

La encuesta "La Defensa Nacional y las Fuerzas Armadas" recoge, entre otras cuestiones, la opinión de los españoles en asuntos relacionados con el sentimiento y compromiso de sentirse español, la percepción de vivir en un mundo inseguro o cuál es la impresión de la sociedad española hacia sus Fuerzas Armadas.

Las encuestas CIS se diseñan para un nivel de confianza del $95,5 \%$ con un error de $\pm 2 \%$ en el conjunto de la muestra ${ }^{2}$.

A través de los datos de las cinco últimas encuestas, desde 2002 hasta 2011, se analiza cuánto ha interiorizado la sociedad española - a lo largo del tiempoalgunos de los aspectos que sistematizan el régimen de los militares: los símbolos nacionales, el orgullo de sentirse español, el espíritu de sacrificio, la disposición permanente para defender a España o la conciencia de la necesidad de mantener unas FAS.

Resulta razonable suponer que para conseguir que dos realidades, distintas a priori, consigan identificarse es necesario que se valoren positivamente.

Por ello, la primera parte analiza cómo valora la sociedad española a sus Fuerzas Armadas (FAS), a través de cuatro aspectos incluidos en la encuesta "La Defensa Nacional y las Fuerzas Armadas":

- Qué opinión tiene la sociedad española de sus Fuerzas Armadas.

- Cómo califican los españoles a las profesiones militares: la de militar de carrera y la de militar profesional de tropa y marinería (MPTM).

- Si animaría a su hijo o hija a hacerse MPTM.

- Cómo valora que la mujer ocupe puestos en las FAS.

En la segunda parte del artículo se explora - a través de otras cuestiones de las encuestas del CIS - cómo se identifican los españoles con valores consustanciales a los miembros de las Fuerzas Armadas.

En la tercera parte se examina el apoyo responsable de la sociedad española para mantener a sus Fuerzas Armadas. Finalmente, se exponen unas conclusiones que resumen las ideas anteriores.

\section{1a PARTE: CÓMO VALORA LA SOCIEDAD ESPAÑOLA SUS FUERZAS ARMADAS}

\subsection{Opinión de los españoles sobre sus FAS}

Desde 2002, la opinión que tiene la sociedad española sobre sus Fuerzas Armadas ha mejorado significativamente en todos los rangos de edad aunque, como se muestra en el gráfico, esta opinión mejora con la edad del individuo.

En 2011, el 62,2\% de los españoles tenía una buena o muy buena opinión de sus Fuerzas Armadas.

Cuando se segrega por CCAA, se observa que, a lo largo de la última década, en el País Vasco, en Navarra o en Cataluña, el colectivo que tiene una buena o muy buena opinión de sus Fuerzas Armadas es más reducido.

Estas tres comunidades se caracterizan porque el porcentaje de nacionalistas es elevado, esto es, de ciudadanos que se sienten únicamente de su comunidad autónoma o más próximos a esta que a España. En concreto supone el 53\% en Navarra, el 38\% en el País Vasco y el 33\% en Cataluña.

Resulta notable que, a pesar del enorme esfuerzo desarrollado estos años para estrechar el contacto de las Fuerzas Armadas con la sociedad civil, el porcentaje de españoles con buena o muy buena opinión de las Fuerzas Armadas se ha mantenido prácticamente constante en el País Vasco y en Cataluña. 

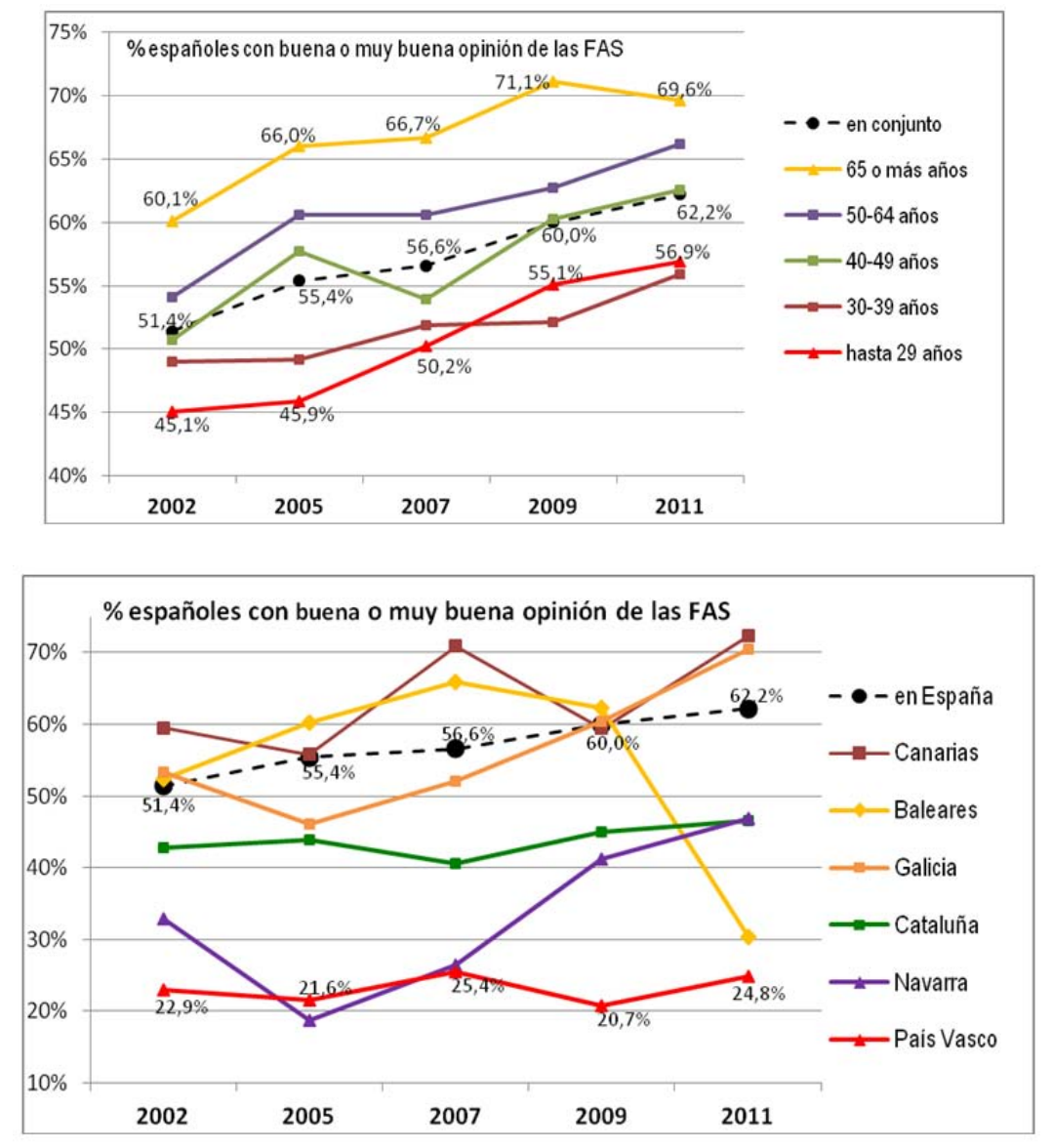

Hay que destacar, además, la brusca disminución en 2011, del colectivo que tiene una buena o muy buena opinión de sus FAS en la Comunidad Illes Balears ${ }^{3}$.

\subsection{Valoración de las profesiones militares}

Al valorar al mismo tiempo varias profesiones, los españoles sitúan en último lugar las profesiones mili- tares, tanto la de militar de carrera como la de soldado o marinero profesional (MPTM).

No obstante, como se aprecia en el gráfico, ambas profesiones militares son las que han tenido una tendencia creciente más acusada a lo largo de la última década.

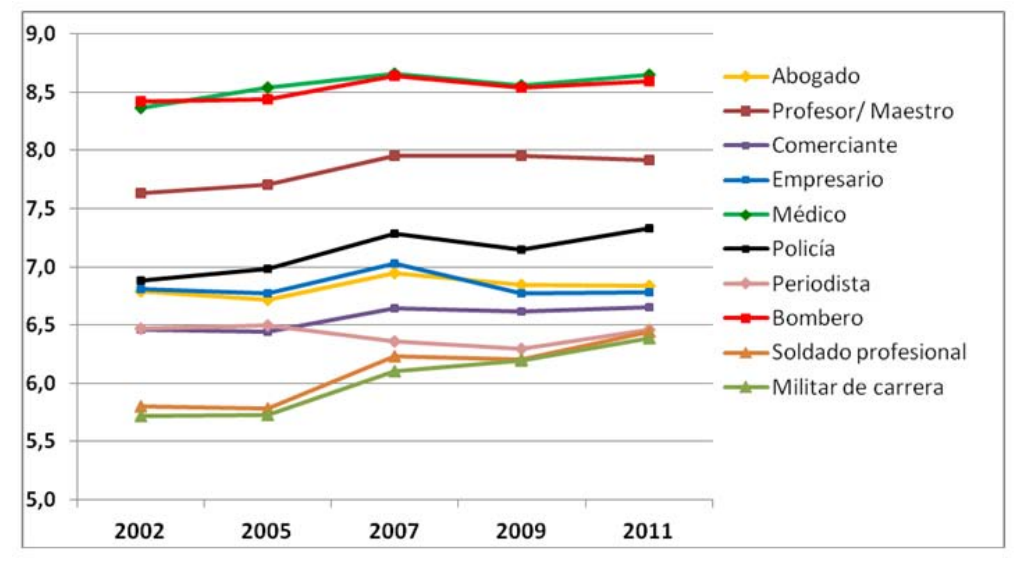


Aunque se valora ligeramente mejor al MPTM que al de militar de carrera, ambas puntuaciones han ido parejas y convergiendo hasta alcanzar un 6,4 en 2011.
Segregando por edades, las valoraciones mejoran con la edad.

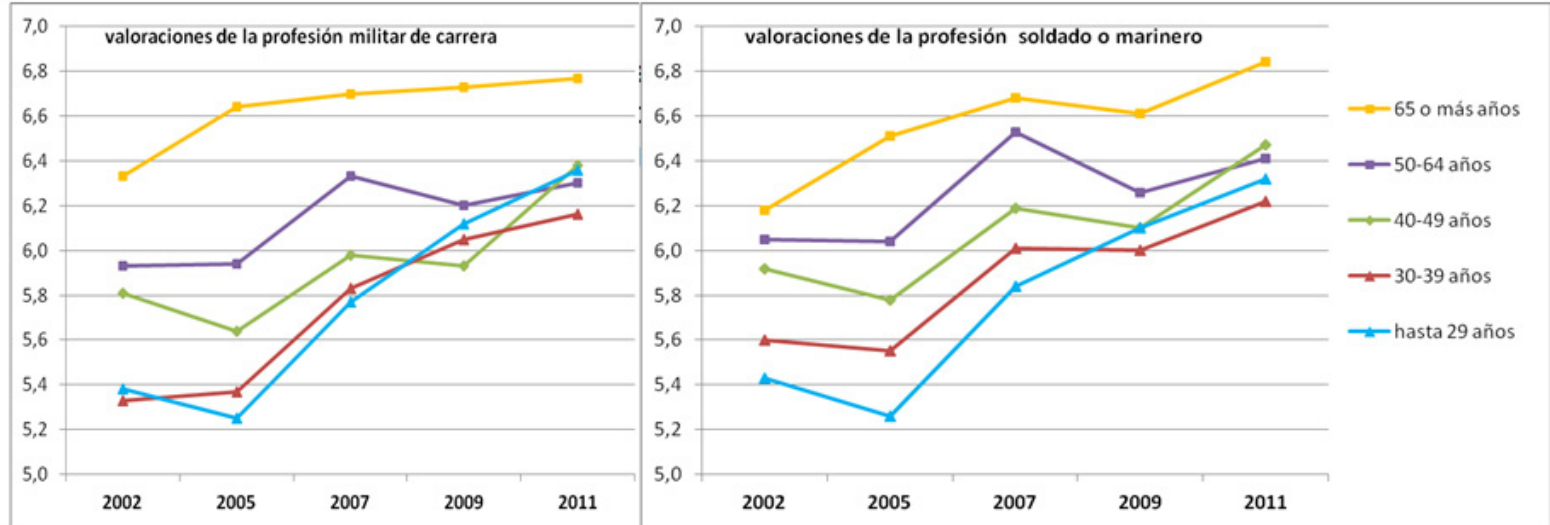

La interpretación es doble: por un lado, la sociedad española no diferencia ambas carreras y por otro lado, la valoración depende de la edad: más alta cuanta mayor edad se tenga y más baja, precisamente, en el colectivo que no ha vivido la milicia obligatoria.

Por otra parte, la semejanza de ambas distribuciones permite inferir que los ciudadanos no distinguen al militar de carrera del MPTM.
En 2011, únicamente los ciudadanos del País Vasco y Navarra suspenden la valoración del militar de carrera aunque con puntuaciones muy próximas al aprobado.

Entre las comunidades más nacionalistas ${ }^{4}$ : País Vasco, Cataluña o Navarra, sus desviaciones típicas ${ }^{5}$ son las más altas lo que apunta a unos colectivos muy heterogéneos donde hay grupos que puntúan muy bajo y otros alto.

\begin{tabular}{|c|c|c|c|c|}
\hline \multirow{2}{*}{ CCAA } & \multicolumn{2}{|c|}{ Militar de carrera } & \multicolumn{2}{c|}{ Soldado o marinero profesional } \\
\cline { 2 - 5 } & $\begin{array}{c}\text { Valoración } \\
\text { media }\end{array}$ & Desviación típica & $\begin{array}{c}\text { Valoración } \\
\text { media }\end{array}$ & Desviación típica \\
\hline Andalucía & $\mathbf{6 , 8 8}$ & 2,43 & $\mathbf{6 , 7 1}$ & 2,53 \\
\hline Aragón & $\mathbf{6 , 4 3}$ & 2,61 & $\mathbf{6 , 6 2}$ & 2,43 \\
\hline Asturias & $\mathbf{6 , 9 5}$ & 2,08 & $\mathbf{6 , 7 8}$ & 1,98 \\
\hline Baleares & $\mathbf{5 , 6 8}$ & 2,79 & $\mathbf{5 , 8 6}$ & 2,49 \\
\hline Canarias & $\mathbf{6 , 9 3}$ & 2,56 & $\mathbf{7 , 0 8}$ & 2,44 \\
\hline Cantabria & $\mathbf{6 , 8 7}$ & 2,19 & $\mathbf{6 , 8 8}$ & 2,14 \\
\hline Castilla La Mancha & $\mathbf{7 , 2 5}$ & 1,94 & $\mathbf{6 , 7 2}$ & 2,02 \\
\hline Castilla y León & $\mathbf{6 , 7 9}$ & 1,96 & $\mathbf{6 , 5 5}$ & 2,09 \\
\hline Cataluña & $\mathbf{5 , 4 4}$ & 2,79 & $\mathbf{5 , 8}$ & 2,87 \\
\hline Valencia & $\mathbf{6 , 5 9}$ & 2,47 & $\mathbf{6 , 8 6}$ & 2,34 \\
\hline Extremadura & $\mathbf{7 , 1 9}$ & 2,44 & $\mathbf{7 , 2 2}$ & 2,3 \\
\hline Galicia & $\mathbf{6 , 6 1}$ & 2,15 & $\mathbf{6 , 5 6}$ & 2,04 \\
\hline Madrid & $\mathbf{6 , 3 7}$ & 2,46 & $\mathbf{6 , 2 7}$ & 2,43 \\
\hline Murcia & $\mathbf{7 , 1 2}$ & 2,14 & $\mathbf{7 , 3 2}$ & 2,02 \\
\hline Navarra & $\mathbf{4 , 9 3}$ & 3,14 & $\mathbf{5 , 2 6}$ & 3,27 \\
\hline País Vasco & $\mathbf{4 , 6 4}$ & 2,7 & $\mathbf{5 , 1 1}$ & 2,8 \\
\hline Rioja & $\mathbf{6 , 7 1}$ & 1,49 & $\mathbf{7 , 1 2}$ & 2,55 \\
\hline
\end{tabular}


Las comunidades que valoran más alto ambas profesiones son Extremadura, Murcia y Castilla La Mancha.

\subsection{Animaría a su hijo a hacerse soldado profesional}

A lo largo de la última década, el porcentaje ${ }^{6}$ que sí animaría a su hijo -en el supuesto de tenerlo- si decidiese ingresar como soldado o marinero profesional ha aumentado, alcanzando, en 2011, el 47\%, mientras que el colectivo que desaconsejaría esa decisión ha permanecido prácticamente constante alrededor del 32\%.

Desde 2007, independientemente de la edad, la tendencia del colectivo que animaría a su hijo hacerse soldado o marinero profesional ha sido creciente.

Las comunidades más nacionalistas son las que más desaconsejarían a sus hijos hacerse soldado o marinero profesional?
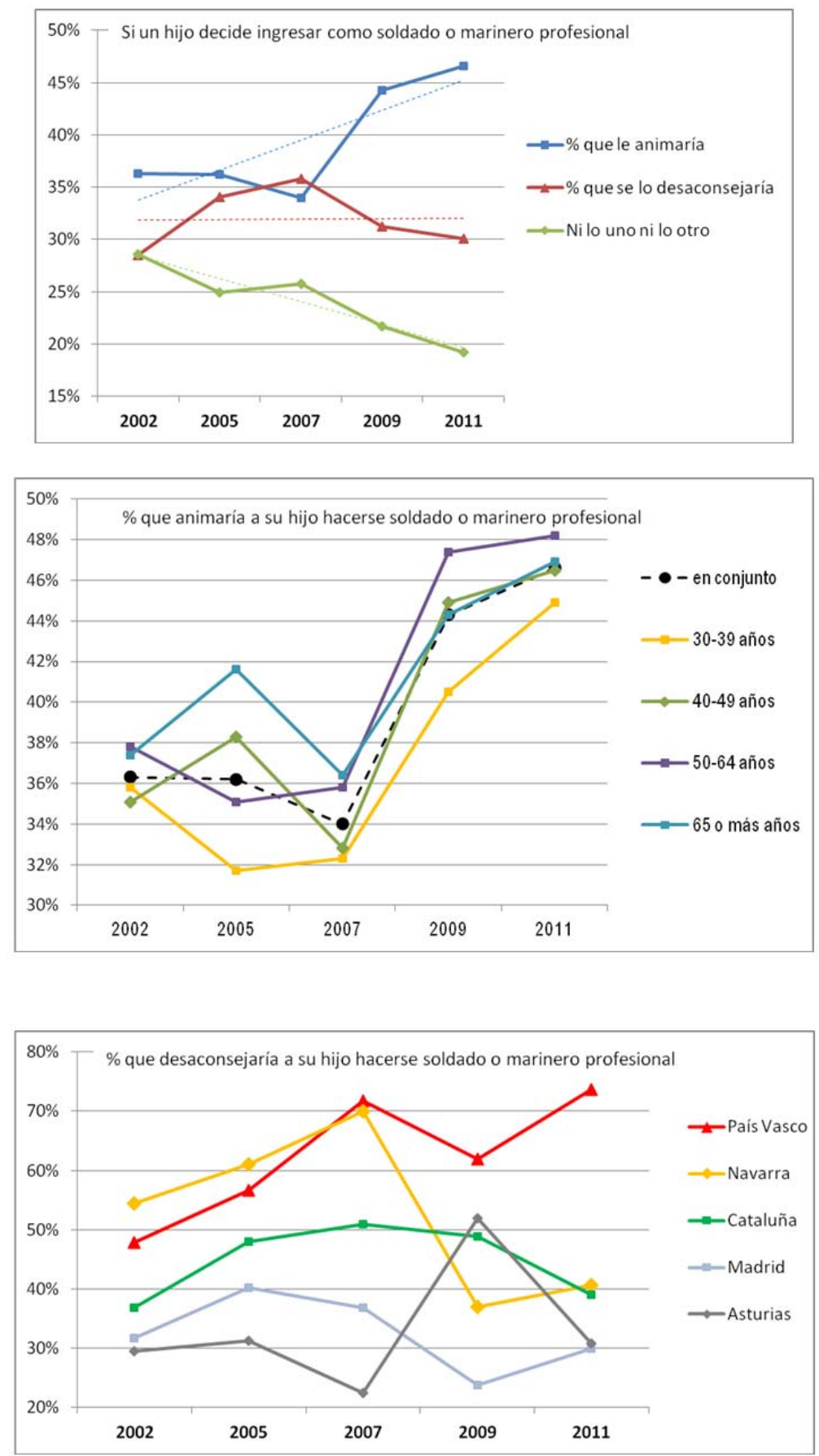
Destaca la disminución desde 2007 en Navarra, del porcentaje que desaconsejaría esta decisión a sus hijos. En la última década, la tendencia de este colectivo solo es significativamente creciente en el País Vasco.

\subsection{Cómo valora que la mujer ocupe puestos en las FAS}

Desde la profesionalización de las Fuerzas Armadas y la consiguiente incorporación de la mujer a estas, la gran mayoría de los españoles, independientemente del sexo, está de acuerdo con que la mujer ocupe puestos de combate. Desde 2007 este porcentaje se mantiene constante rozando el $90 \%$.
Este reconocimiento mayoritario, su tendencia creciente y, sobre todo, que no haya diferencias por sexo, permite concluir que la sociedad española es plenamente madura con la integración de la mujer en las Fuerzas Armadas.

La edad influye en esta respuesta: cuanto más joven, mayor es el colectivo que está de acuerdo que la mujer ocupe puestos de combate. Sin embargo, con el paso de los años estas diferencias se acortan y constituyen otro rasgo de normalidad en la relación mujer y FAS como se aprecia en el gráfico.

La respuesta también depende de la comunidad autónoma aunque también, con el transcurso de los años, estas diferencias se han reducido ${ }^{8}$.
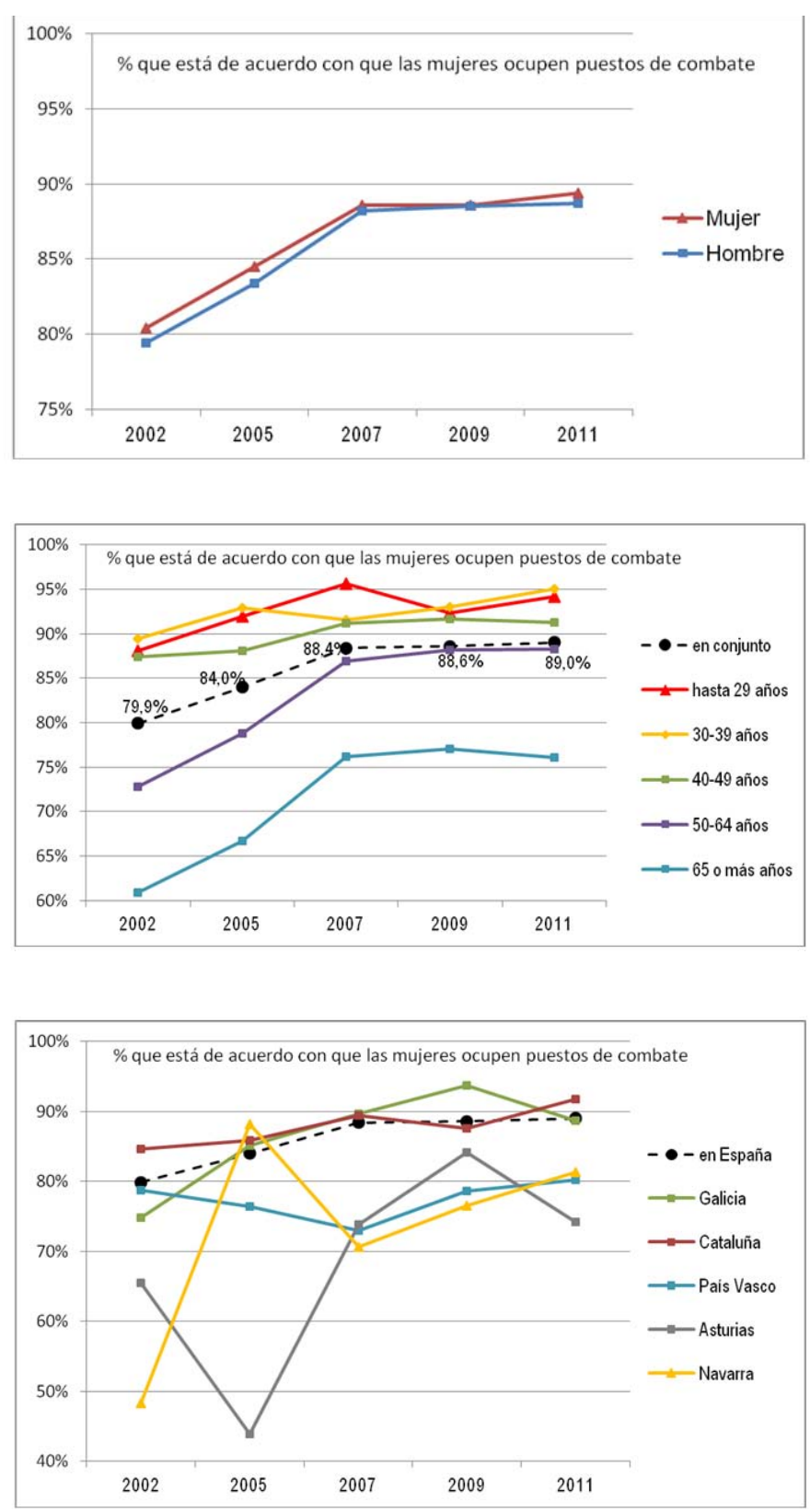


\section{2a PARTE: IDENTIFICACIÓN SOCIEDAD ESPAÑOLA - FUERZAS ARMADAS}

Para estimar, a través de las encuestas del CIS, el grado con que los españoles se identifican con sus Fuerzas Armadas, se analiza cuánto asume la sociedad española ciertos aspectos característicos e intrínsecos de los militares: la emoción por los símbolos nacionales, el orgullo de sentirse español, el espíritu de sacrificio o la disposición permanente para defender a España.
Aspectos que, por su importancia, se recogen en las Reales Ordenanzas (RROO) para las Fuerzas Armadas que son las normas que establecen el comportamiento, derechos y deberes del militar español ${ }^{9}$.

\subsection{Orgullo de ser español}

En conjunto, más del $80 \%$ de la sociedad española siente mucho o bastante orgullo de ser español y como se observa en el gráfico, este sentimiento crece con la edad.

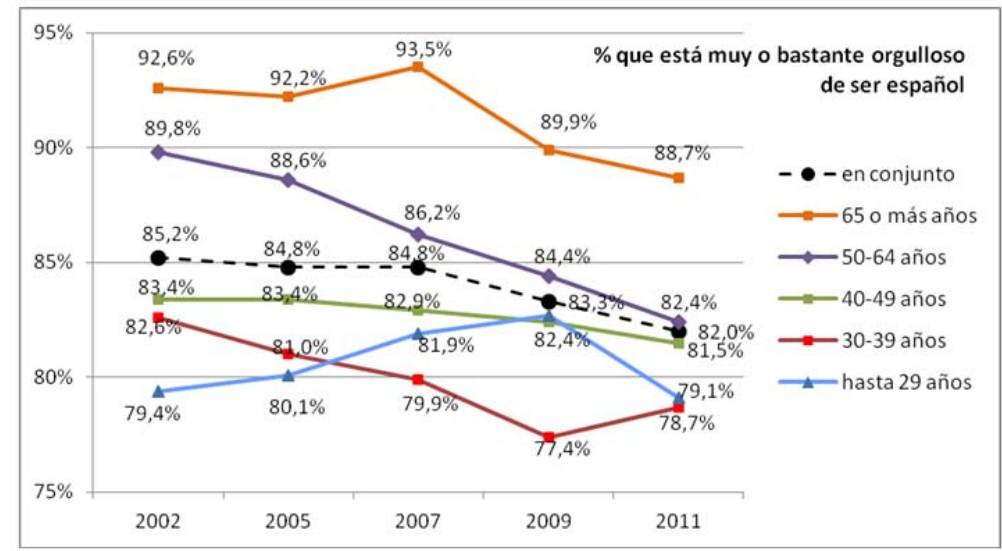

Sin embargo, en la última década, el sentimiento de orgullo está disminuyendo y lo hace más rápido precisamente en el colectivo con mayor sentimiento de orgullo.

La comunidad autónoma también influye en el sentimiento de orgullo de ser español. Las menos orgullosas son, justamente, las más nacionalistas ${ }^{10}$ excepto Canarias ${ }^{11}$.
Concretamente, el porcentaje de ciudadanos que, en 2011, se declara mucho o bastante orgulloso de ser español alcanza el $44 \%$ en el País Vasco, el $47 \%$ en Navarra o el $61 \%$ en Cataluña.

Hay, sin embargo, otras diez CCAA -que se muestran en el siguiente gráfico- donde el porcentaje que siente mucho o bastante orgullo de ser español alcanza en 2011 , al menos, el $90 \%{ }^{12}$.

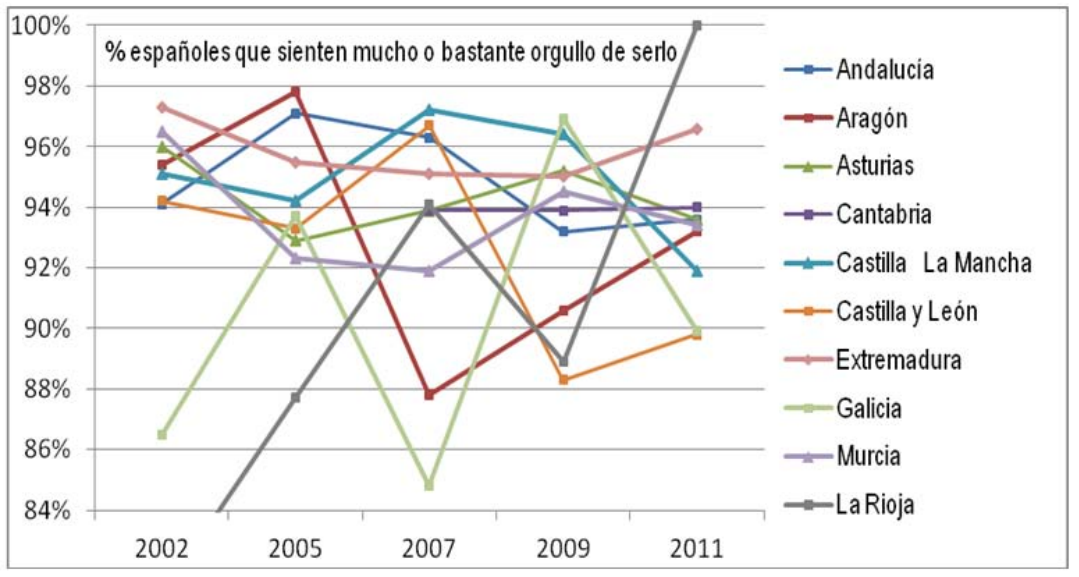




\subsection{Símbolos nacionales}

El artículo sexto de las RROO para las Fuerzas Armadas, expresa que todo militar mostrará el máximo respeto a la Bandera de España e Himno Nacional como símbolos de la Patria transmitidos por la historia.
Los siguientes gráficos muestran cómo ha sido la evolución por CCAA $^{13}$ de los colectivos que apenas se sienten orgullosos de ser español o que muestran poca o ninguna emoción ante la bandera, el himno o en los actos castrenses.

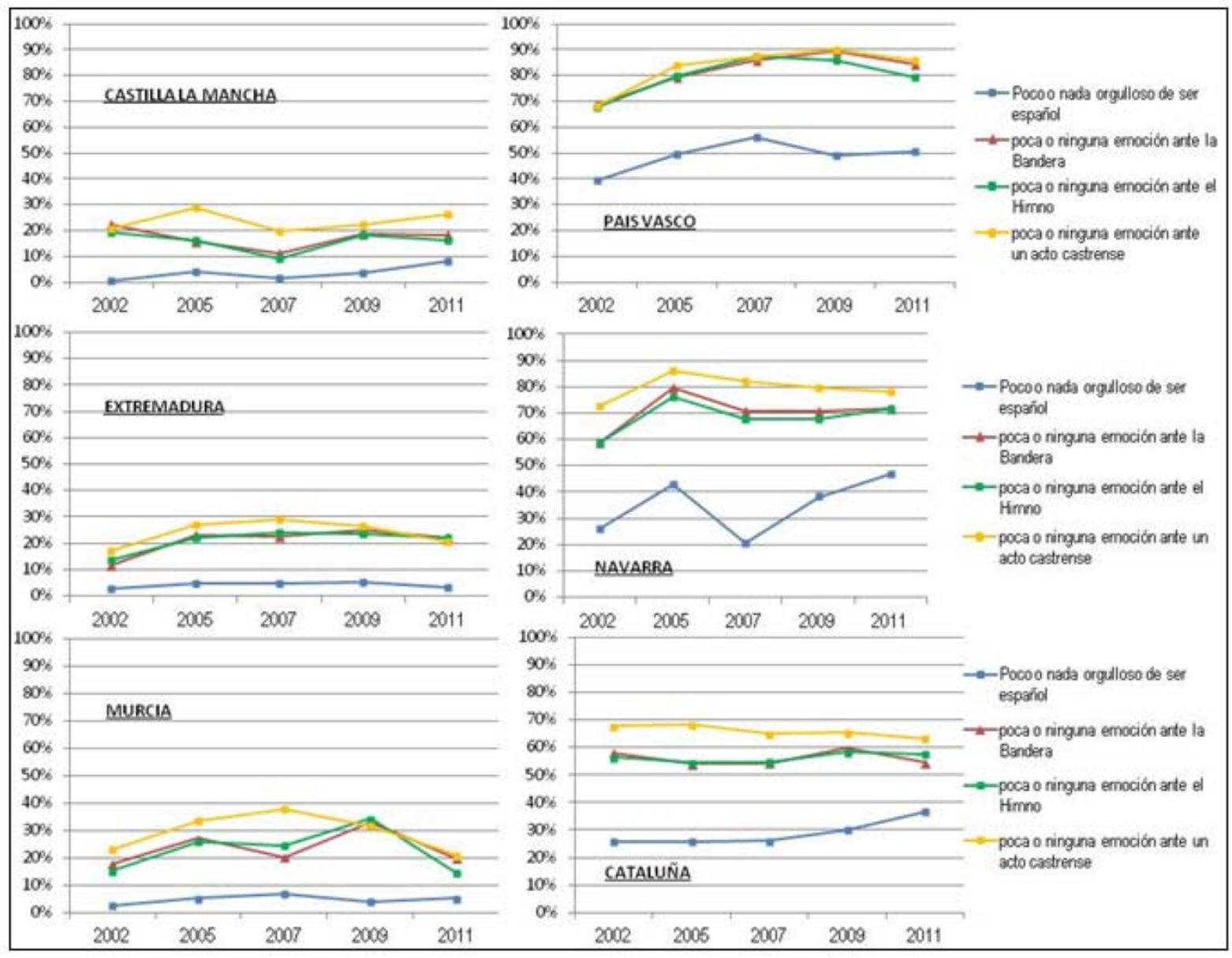

Su examen permite concluir lo siguiente:

- Los ciudadanos que se emocionan con los símbolos o en los actos castrenses (en el gráfico los que no se emocionan) varían mucho con la comunidad autónoma: cuanto más nacionalista, menor es la emoción.

- Independientemente de la comunidad autónoma, los actos castrenses emocionan menos que la bandera o el himno.

- Los españoles, independientemente de su comunidad autónoma, no identifican el orgullo de ser español con la emoción que provocan los símbolos que lo representan: hay más población orgullosa de ser española que de emocionarse con la bandera o el himno. Sin embargo, esta disociación es mayor cuanto más nacionalista es la comunidad.

\subsection{Espíritu de sacrificio}

En la encuesta CIS, se pregunta si, aparte de la familia, considera que hay algo que merezca la pena sacrificarse.

Hay un menor sentido del sacrificio entre las mujeres que entre los hombres, si bien esta diferencia es pequeña: en 2011 , el $49 \%$ de las mujeres no se sacrificaría frente al $43 \%$ de los hombres. 


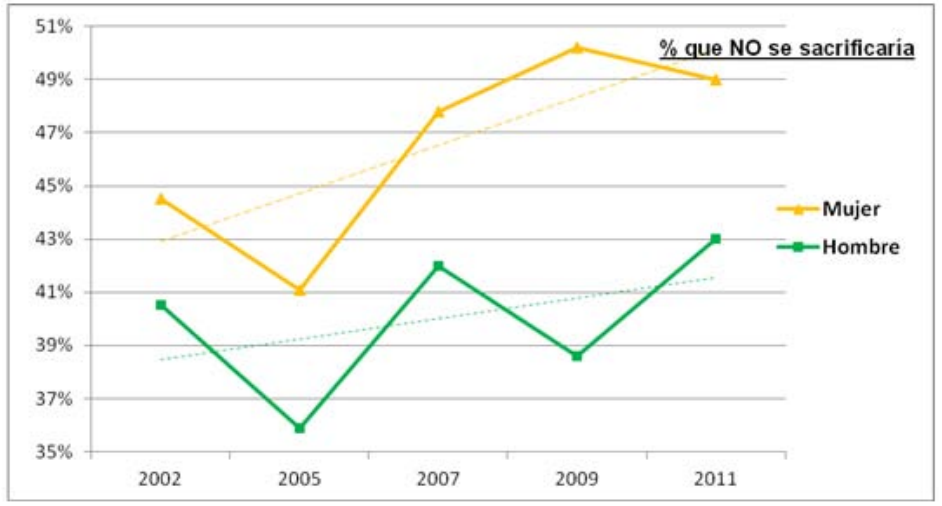

Más significativo resulta que el colectivo que no se sacrificaría está aumentando y algo más rápido entre las mujeres.
Si se segrega por CCAA, destacan las siguientes comunidades donde la predisposición del colectivo que no se sacrificaría ha aumentado más en la última década:

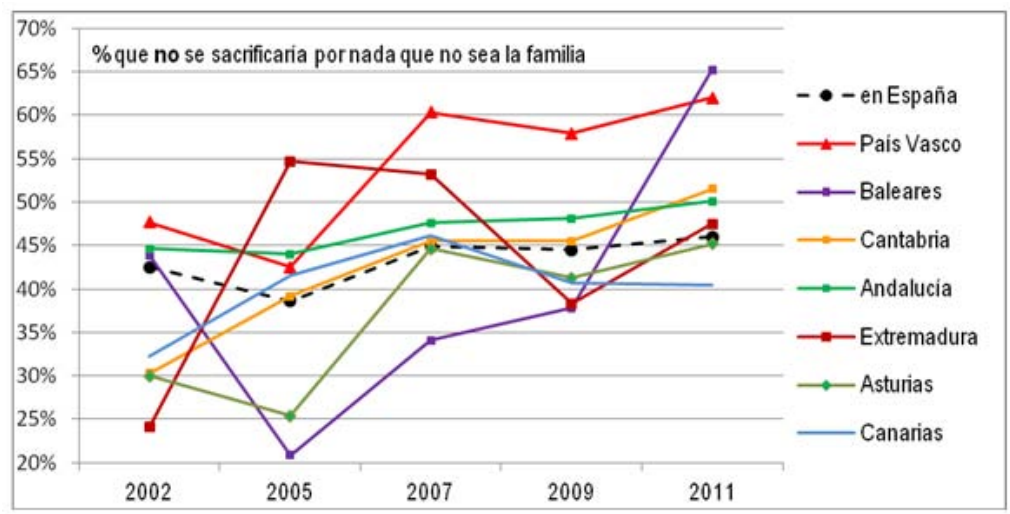

Esta tendencia a no sacrificarse es independiente de la edad como se observa en el siguiente gráfico donde, en todos los rangos de edad, los que creen que sí merece la pena sacrificarse por algo está disminuyendo.

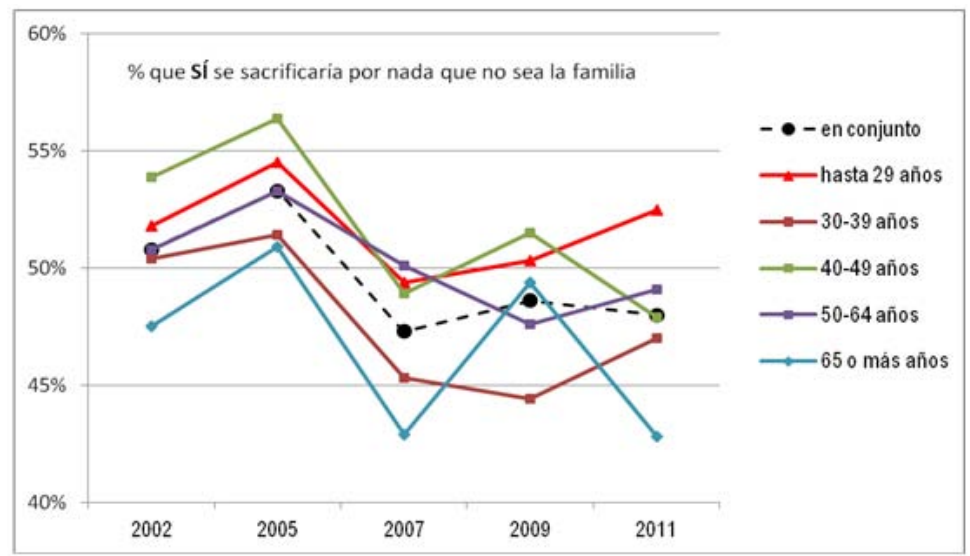




\subsection{Disposición permanente para defender a España}

La Constitución Española establece, en su artículo 30.1, que los españoles tienen el derecho y el deber de defender a España.
Se analiza el porcentaje de españoles que participaría voluntariamente en la defensa de España en caso de ataque militar ${ }^{14}$.

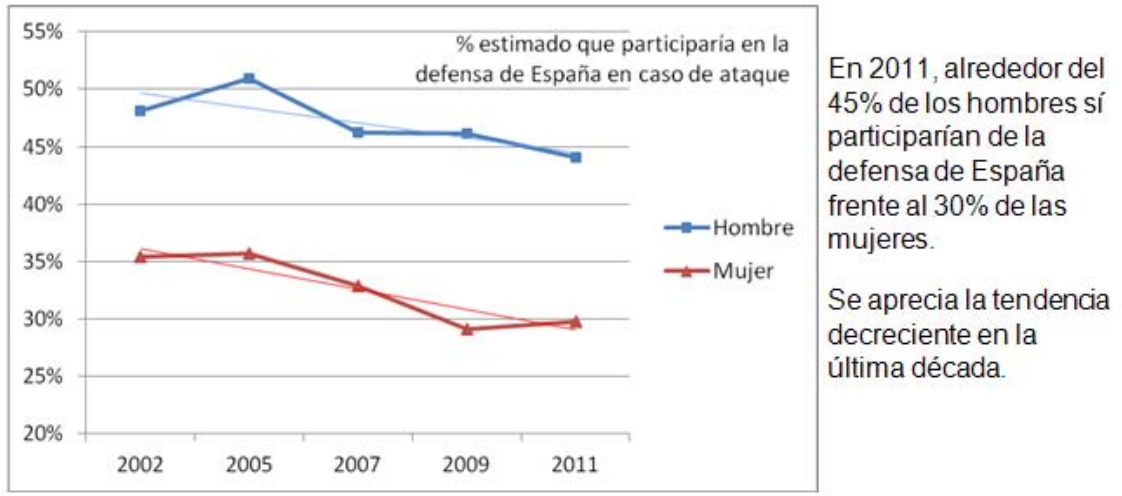

Tendencia decreciente que es independiente de la población cuya aptitud era más positiva (en el rango edad y que es más acusada, precisamente, entre la de 40 a 64 años):

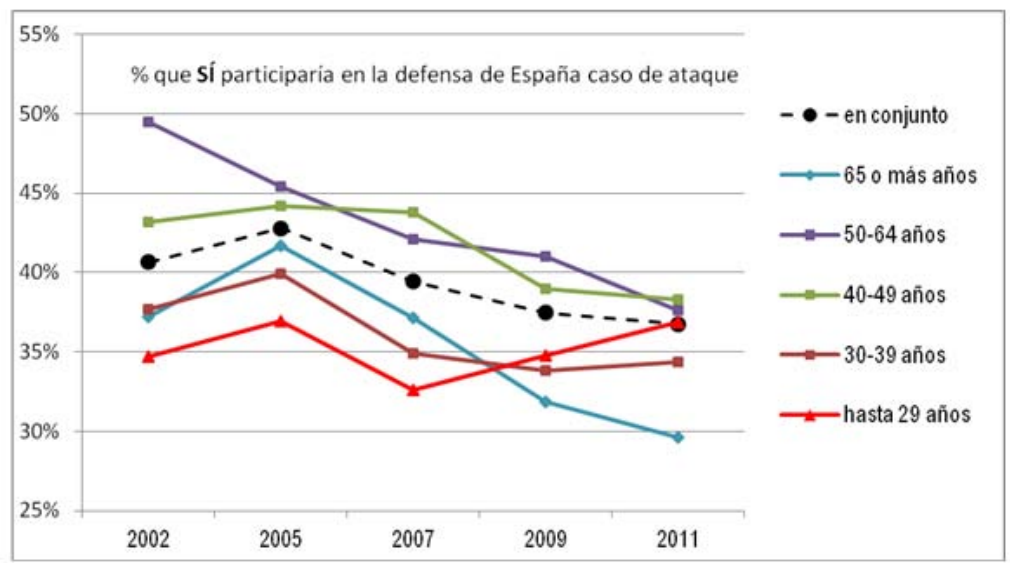

Si se segrega por CCAA, destacan las siguientes comunidades donde la tendencia del colectivo que sí participaría de la defensa de España ha disminuido más en la última década. Se incluye, además, el País Vasco por tener el porcentaje más reducido:

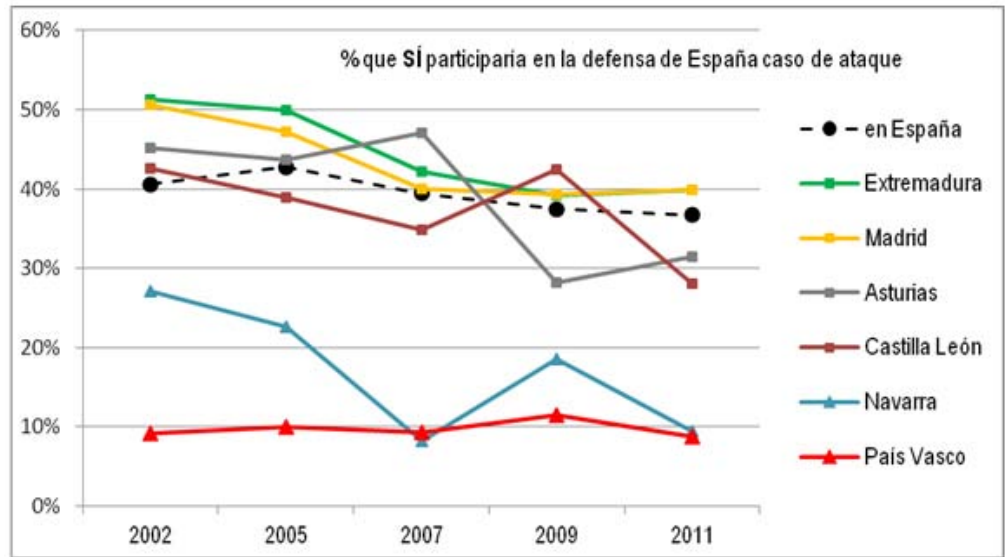




\section{3a PARTE: CONCIENCIA DE LA NECESIDAD DE MANTENER UNAS FAS}

Para conocer si la sociedad española siente, de forma reflexiva, la necesidad de mantener sus Fuerzas Armadas, se analizan las siguientes cuestiones planteadas en las encuestas del CIS:

- ¿Existe algún país que represente una amenaza militar contra España?
- ¿Son necesarios los ejércitos?

- ¿Considera insuficiente el presupuesto de defensa?

\section{1. ¿Existen países que representen una amenaza militar contra España?}

Desde 2007, la percepción de que existen países que suponen una amenaza está disminuyendo, independientemente del sexo y de la edad. En 2011 ronda el $30 \%$ de la población.
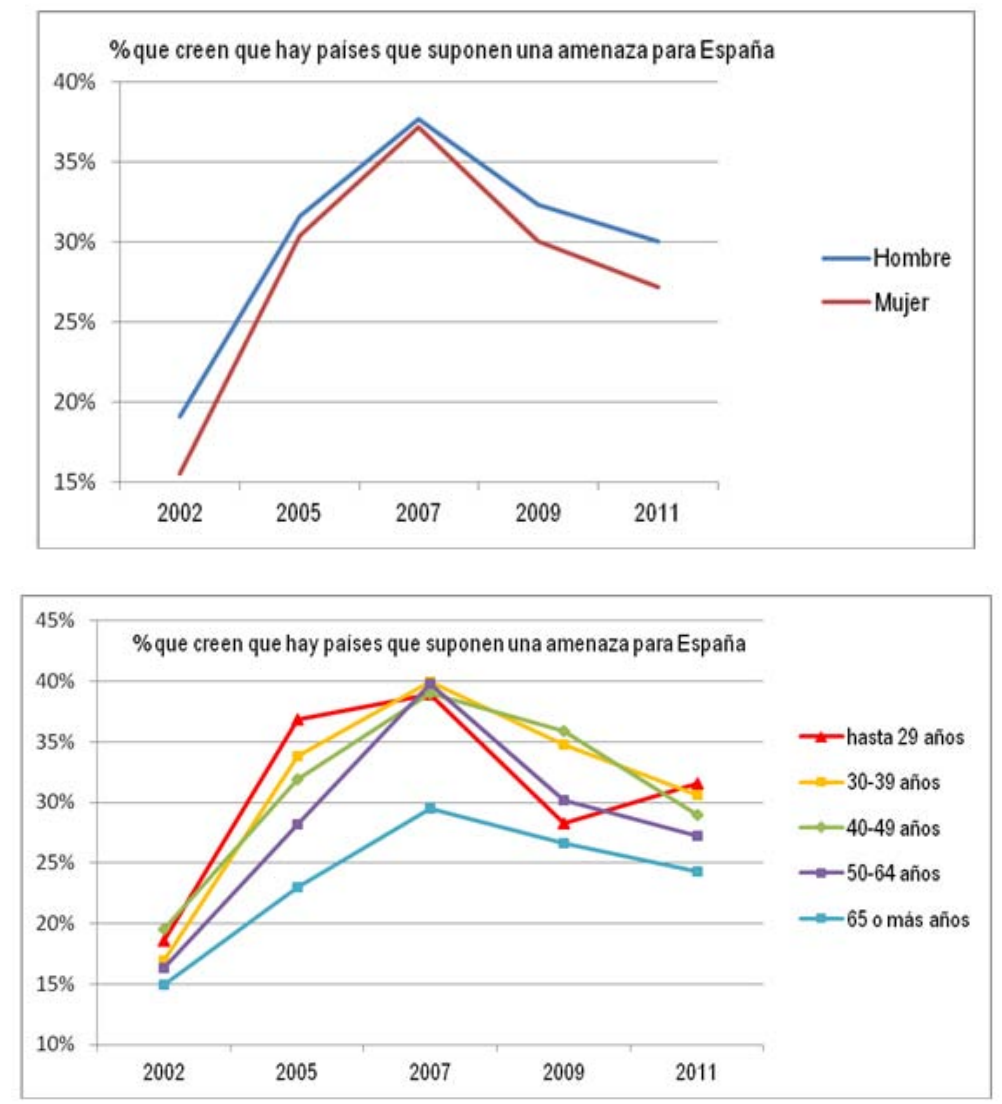

La percepción mayoritaria en la sociedad española es que cada vez hay menos amenazas militares contra España. Y esto tiene reflejo en la siguiente cuestión.

\section{2. ¿Son necesarios los ejércitos?}

La mayoría, independientemente del sexo, considera que los ejércitos seguirán siendo necesarios ${ }^{15}$ aunque, desde 2009, el porcentaje ha disminuido ligeramente.

Si se discrimina por la edad, se observa que esta tendencia decreciente desde 2009, ocurre en todas las edades excepto para los mayores de 65 años.

\section{3. ¿Considera insuficiente el presupuesto de defensa?}

El colectivo más numeroso - y que permanece invariable a lo largo de los años en torno al $30 \%$ - es el que está de acuerdo con los recursos asignados para la defensa nacional ${ }^{16}$.

Destaca en 2011 el aumento de la población que lo considera excesivo hasta el $26 \%$.

En relación con la pregunta -añadida en 2009- de si está de acuerdo en destinar más presupuesto para mantener el ejército profesional y las Fuerzas Arma- 

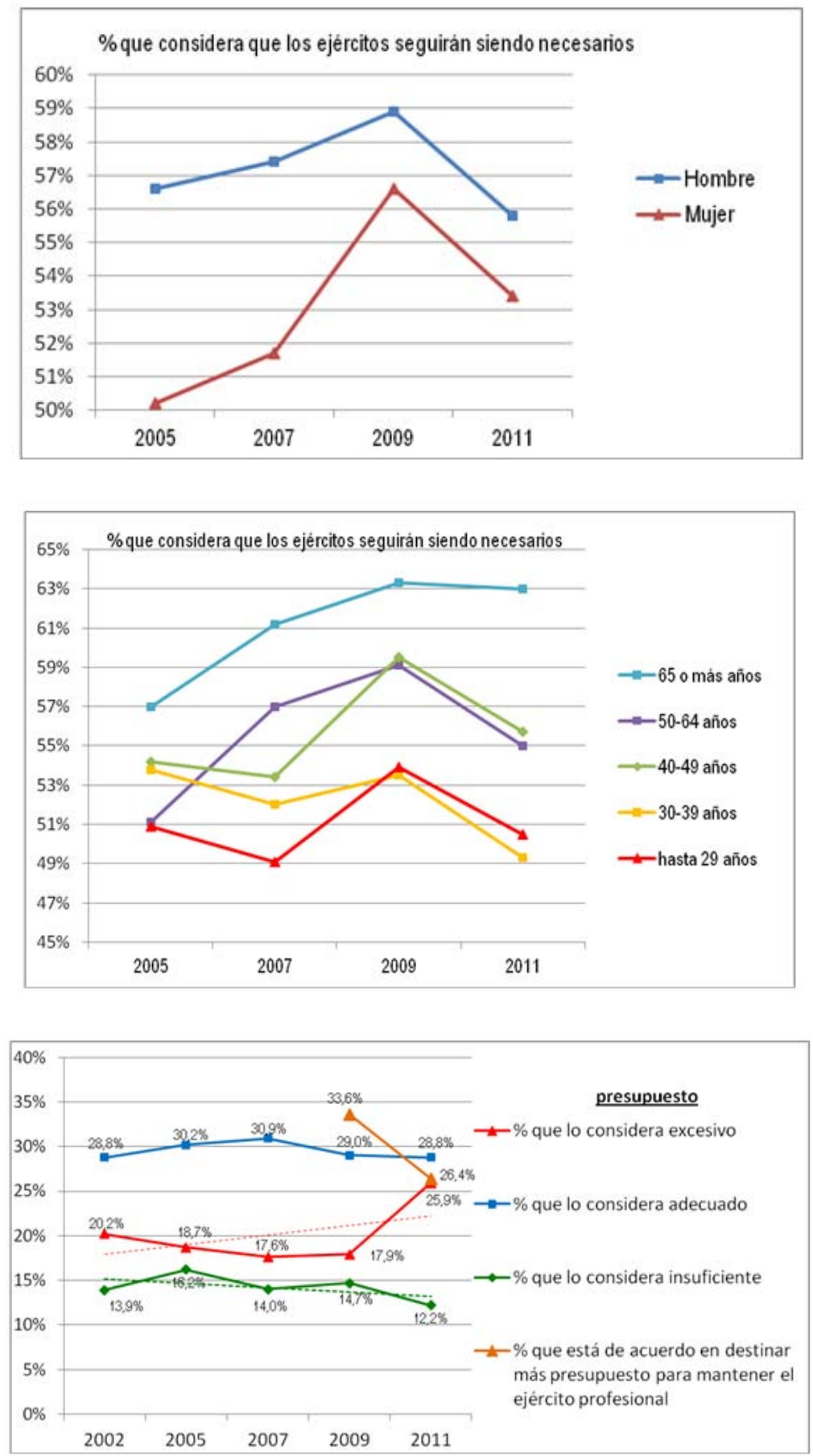

das, se aprecia una brusca disminución en 2011, coherente con lo mencionado anteriormente.

El porcentaje que apoyaría un aumento del presupuesto para defensa y que correspondería a las respuestas que consideran insuficiente el presupuesto, es muy reducido, alrededor del $13 \%$ en la última década.

\section{CONCLUSIONES}

La serie de encuestas CIS "La Defensa Nacional y las Fuerzas Armadas" realizadas desde 1997, permite analizar cómo ha evolucionado la valoración de los españoles hacia sus Fuerzas Armadas. 
La sociedad española en su conjunto, especialmente entre los ciudadanos de más edad, valora positivamente a sus Fuerzas Armadas y, en la última década, se percibe un acercamiento entre la sociedad española y sus Fuerzas Armadas que se manifiesta en los siguientes indicadores:

- La opinión sobre las FAS es buena y aumenta de forma constante desde $20022^{17}$. Actualmente, el porcentaje alcanza el $62 \%$.
No obstante, esta opinión no es homogénea: es mejor entre los mayores de 50 años y peor, en general, entre las CCAA más nacionalistas ${ }^{18}$.

- El apoyo a los hijos que deciden ingresar como militares profesionales está aumentando, especialmente desde 2007 y alcanza en 2011 el $47 \%$ que supone diez puntos porcentuales más que en 1999.

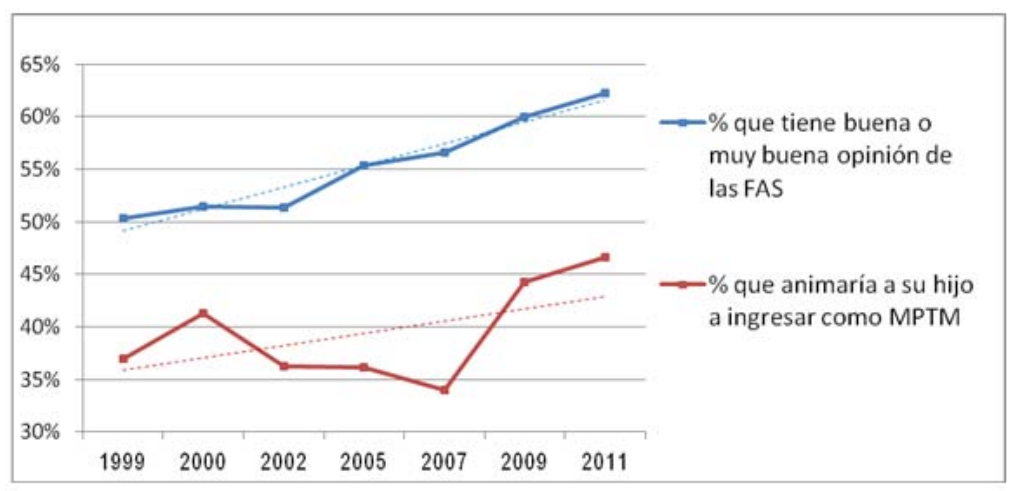

Ambas variables están correlacionadas como se aprecia en sus tendencias.

- La incorporación de la mujer en las Fuerzas Armadas ha sido, desde las primeras encuestas, muy apoyada por la sociedad española pero, con los años, se ha consolidado de tal manera que puede considerarse una asignatura superada.

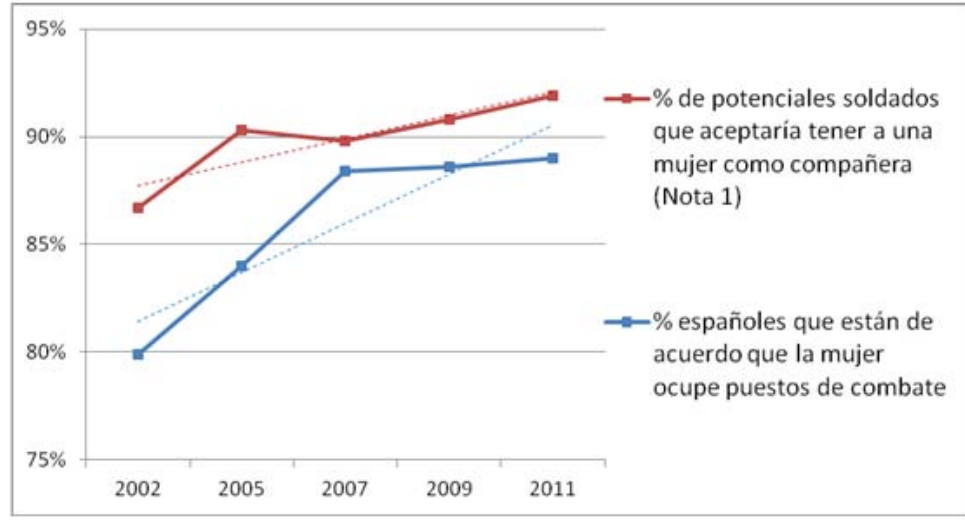

Nota: Para el cálculo del porcentaje en 2011 no se han contabilizado las respuestas NS/NC

En relación con el grado y evolución con que los españoles comparten ciertos aspectos intrínsecos de los miembros de las Fuerzas Armadas, los datos de las encuestas $\mathrm{CIS}$ revelan que:

- El espíritu de sacrificio o la disposición para defender España en caso de un ataque militar no son valores atractivos para la sociedad española y su tendencia es decreciente.

- El porcentaje de españoles que sienten mucho o bastante orgullo de serlo es elevado: hoy se estima en el $82 \%$. No obstante, está disminuyendo con los años y lo hace ligeramente más rápido en- 


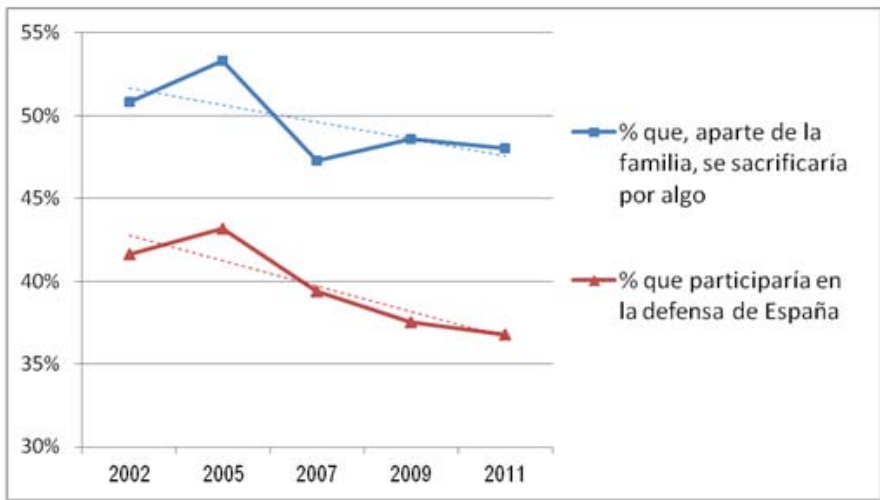

tre el colectivo mayor de 50 años que es, precisamente, el que siente más orgullo.

- Los españoles no asocian el orgullo de ser español con la emoción que provocan los símbolos que lo representan: todos los que se emocionan con la bandera se sienten orgullosos de ser españoles pero la inversa no es cierta. Además esta disociación es mayor cuanto más nacionalista es la comunidad.

Este desacuerdo se señala con flechas en el siguiente gráfico, donde prácticamente uno de cada cuatro españoles que declara sentirse español, no le emociona la bandera de España ${ }^{19}$.

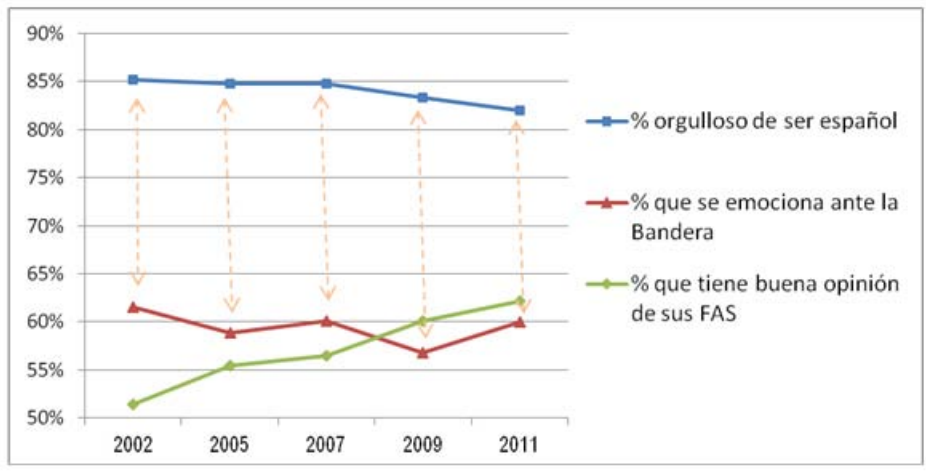

En cuanto a la inclinación que manifiesta la sociedad española para mantener a sus Fuerzas Armadas, el siguiente gráfico permite, a través de tres aspectos, inferir estos apoyos:

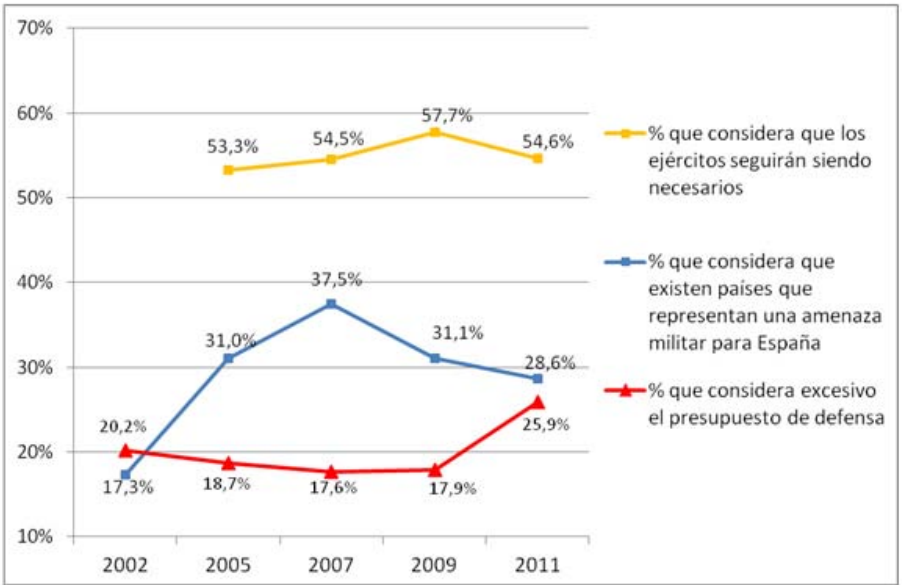


- Aproximadamente, el 55\% de los españoles considera que los ejércitos, con su modelo y funciones actuales, siguen siendo necesarios para la defensa, porcentaje que permanece prácticamente constante a lo largo de los años.

- Desde 2007, la disminución del colectivo que percibe que hay países que representan una amenaza militar podría explicar el aumento del número de españoles que considera excesivo el presupuesto de defensa que alcanzó en 2011 el 26\%.

La característica más notable al estudiar la serie de encuestas CIS ha sido la enorme heterogeneidad que existe en la sociedad española cuando se segmenta por su edad, por su comunidad autónoma o por su ideología política, aspecto este que no se ha sido tratado en el artículo.

El análisis conjunto de los datos muestra una sociedad española con una elevada opinión de sus Fuerzas Armadas pero con un menor compromiso hacia los valores que estas representan y con un creciente interés para que su presupuesto se ajuste al menor presentimiento de amenazas militares contra España.

\section{NOTAS}

1 La última encuesta se realizó entre septiembre y octubre de 2011.

2 Se puede afirmar estadísticamente que, de 100 encuestas realizadas independientemente, en 95,5 de ellas, los porcentajes conseguidos son extrapolables a la población española con un margen de error del $\pm 2 \%$.

3 En la encuesta de 2011 otros indicadores muestran un crecimiento muy acusado del nacionalismo en Baleares.

4 Se emplea la expresión nacionalista para indicar que los ciudadanos se sienten únicamente de su comunidad autónoma o más próximos a esta que a España.

5 La desviación típica es una medida de dispersión e indica cuánto se alejan los valores respecto al promedio (media).

6 La pregunta fue hecha solo a los ciudadanos mayores de 25 años y, en 2009, se amplió a los mayores de 28 años.

7 Se ha añadido la Comunidad de Madrid y la del Principado de Asturias para permitir la comparación.
8 Se han representado solo algunas CCAA.

9 Real Decreto 96/2009, de 6 de febrero.

10 Se emplea la expresión nacionalista para indicar que los ciudadanos se sienten únicamente de su comunidad autónoma o más próximos a esta que a España.

11 Que, con un 38\% de nacionalistas, el $83 \%$ se siente mucho o bastante orgulloso de ser español.

12 Es necesario ser prudente al analizar los cambios bruscos en los resultados en La Rioja que se interpretan porque la muestra de esa comunidad es la más reducida (17) y no representa fielmente a su población que, seguramente, posee una variabilidad de opiniones mucho mayor que la que puede recoger la propia muestra.

13 Se han escogido las CCAA más extremas a lo largo de los últimos años.

14 Para estimar el porcentaje que participaría voluntariamente en la defensa de España se ponderaron las respuestas (1 para "sí, con toda seguridad"; 0,8 para "probablemente sí" y 0,05 para "probablemente no").
15 Las otras opciones para el futuro de los ejércitos eran su desaparición, su transformación en una policía internacional o bien dedicada exclusivamente a ayuda humanitaria.

16 Lo que falta en cada año para llegar al $100 \%$ es el porcentaje que corresponde a las respuestas NS/NC.

17 Coincide con la participación de España en misiones en Oriente Medio tras el 11-S: la misión en Iraq comenzó en 2003 y los resultados pudieron ser recogidos en la encuesta CIS de 2005.

18 Se emplea la expresión nacionalista para indicar que los ciudadanos se sienten únicamente de su comunidad autónoma o bien más próxima a esta que a España.

19 Este valor es para el conjunto de la población española. Cuando se discrimina por comunidades autónomas, los porcentajes varían mucho. 